PROCEEDINGS OF THE

AMERICAN MATHEMATICAL SOCIETY

Volume 133, Number 11, Pages 3341-3344

S 0002-9939(05)07909-8

Article electronically published on May 9, 2005

\title{
DISCRETE SPECTRA OF $C^{*}$-ALGEBRAS AND ORTHOGONALLY CLOSED SUBMODULES IN HILBERT $C^{*}$-MODULES
}

\author{
MASAHARU KUSUDA
}

(Communicated by David R. Larson)

\begin{abstract}
Let $A$ and $B$ be $C^{*}$-algebras and let $X$ be an $A$ - $B$-imprimitivity bimodule. Then it is shown that if the spectrum $\widehat{A}$ of $A$ (resp. $\widehat{B}$ of $B$ ) is discrete, then every closed $A$ - $B$-submodule of $X$ is orthogonally closed in $X$, and conversely that if $\widehat{A}$ (resp. $\widehat{B}$ ) is a $T_{1}$-space and if every closed $A$ - $B$ submodule of $X$ is orthogonally closed in $X$, then $\widehat{A}$ (resp. $\widehat{B}$ ) is discrete.
\end{abstract}

\section{INTRODUCTION}

Let $A$ be a $C^{*}$-algebra and let $X$ be a Hilbert $A$-module with an $A$-valued inner product $\langle\cdot, \cdot\rangle$. For any closed Hilbert $A$-submodule $Y$ of $X$, we denote by $Y^{\perp}$ the orthogonally complemented subspace of $Y$ in $X$, i.e.,

$$
Y^{\perp}=\{x \in X \mid\langle x, y\rangle=0 \text { for all } y \in Y\} .
$$

We say that a closed $A$-submodule $Y$ of a Hilbert $A$-module $X$ is orthogonally complemented in $X$ if $X$ coincides with $Y \oplus Y^{\perp}$, and that a closed $A$-submodule $Y$ of a Hilbert $A$-module $X$ is orthogonally closed in $X$ if $Y^{\perp \perp}=Y$. If $Y$ is orthogonally complemented in $X$, then it is orthogonally closed in $X$. But the converse is not necessarily true in general. Suppose that $X$ is a full (right) Hilbert $A$-module. Then Schweitzer [7] Theorem 1] has shown that every closed right $A$-submodule of $X$ is orthogonally closed if and only if every closed right $A$-submodule of $X$ is orthogonally complemented in $X$. Here we remark that every full right Hilbert $A$-module is a $\mathcal{K}(X)$-A-imprimitivity bimodule, where $\mathcal{K}(X)$ is the $C^{*}$-algebra generated by those operators $\theta_{x, y}$ with all $x, y \in X$ defined by $\theta_{x, y}(z)=x \cdot\langle y, z\rangle$ for all $z \in X$.

Let $A$ and $B$ be $C^{*}$-algebras and let $X$ be an $A$-B-imprimitivity bimodule. Note that $A$ is isomorphic to $\mathcal{K}(X)$. In this paper, we show that every closed $A$ - $B$ submodule of $X$ is orthogonally closed if and only if every closed $A$ - $B$-submodule of $X$ is orthogonally complemented in $X$. As a corollary, we show that if the spectrum $\widehat{A}$ of $A$ (resp. $\widehat{B}$ of $B$ ) is discrete, then every closed $A$ - $B$-submodule of $X$ is orthogonally closed in $X$, and conversely that if $\widehat{A}$ (resp. $\widehat{B}$ ) is a $T_{1}$-space and if every closed $A$-B-submodule of $X$ is orthogonally closed in $X$, then $\widehat{A}$ (resp. $\widehat{B}$ ) is discrete.

Received by the editors December 3, 2003 and, in revised form, June 23, 2004.

2000 Mathematics Subject Classification. Primary 46L05, 46L08.

Key words and phrases. Hilbert $C^{*}$-modules, orthogonally closed.

(C)2005 American Mathematical Society Reverts to public domain 28 years from publication 


\section{Results}

Let $A$ and $B$ be $C^{*}$-algebras and let $X$ be an $A$ - $B$-imprimitivity bimodule (see [4] or [6] for the definition of an imprimitivity bimodule). We denote by ${ }_{A}\langle\cdot, \cdot\rangle$ the $A$-valued inner product on $X$ as a left Hilbert $A$-module and by $\langle\cdot, \cdot\rangle_{B}$ the $B$-valued inner product on $X$ as a right Hilbert $B$-module, respectively.

Throughout this paper, by an $A$-B-submodule we mean a left $A$-submodule and right $B$-submodule.

Two $C^{*}$-algebras $A$ and $B$ are said to be Morita equivalent if there exists an $A$ - $B$-imprimitivity bimodule. We remark that in this paper, Morita equivalence means strong Morita equivalence in the sense of Rieffel (cf. [6, Remark 3.15]).

Let $A$ be a $C^{*}$-algebra and let $I$ be a closed ideal of $A$. Throughout this paper, unless otherwise stated, by an ideal of $A$ we always mean a two-sided ideal. Then $I^{\perp}$ is defined by

$$
I^{\perp}=\{a \in A \mid a x=0 \text { for all } x \in I\} .
$$

It is easily seen that $I^{\perp}$ is a closed ideal of $A$. Note here that $A$ can be regarded as an $A$ - $A$-imprimitivity bimodule, for the bimodule structure given by the multiplication in $A$, with ${ }_{A}\langle a, b\rangle=a b^{*}$ and $\langle a, b\rangle_{A}=a^{*} b$ for $a, b \in A$ (see [6. Example 3.5]). Then $I$ is a closed $A$ - $A$-submodule of $A$.

Let $A$ and $B$ be $C^{*}$-algebras and let $X$ be an $A$ - $B$-imprimitivity bimodule. For a closed $A$ - $B$-submodule $Y$ of $X$, its orthogonally complemented subspace $Y^{\perp}$ in $X$ is defined by

$$
Y^{\perp}=\left\{x \in X \mid{ }_{A}\langle x, y\rangle=\langle x, y\rangle_{B}=0 \text { for all } y \in Y\right\} .
$$

Lemma 2.1. Let $A$ be a $C^{*}$-algebra and let $I$ be any closed ideal $I$ of $A$. If every closed ideal of $A$ is orthogonally closed in $A$, then we have $A=I \oplus I^{\perp}$.

Proof. Using the fact that for an ideal $J$ of $A$ we have $J \cap J^{\perp}=\{0\}$, it follows that

$$
\left(I \oplus I^{\perp}\right)^{\perp}=I^{\perp} \cap\left(I^{\perp}\right)^{\perp}=\{0\} .
$$

Hence, using the hypothesis, we have $I \oplus I^{\perp}=\left(I \oplus I^{\perp}\right)^{\perp \perp}=A$.

Now we recall the Rieffel correspondence (see [6] Theorem 3.22]). Let two $C^{*}$ algebras $A$ and $B$ be Morita equivalent and let $X$ be an $A$ - $B$-imprimitivity bimodule. We denote by $\mathcal{I}(A)$ (resp. $\mathcal{I}(B)$ ) the set of all closed (two-sided) ideals of $A$ (resp. $B$ ), and by $\mathcal{S}(X)$ the set of closed $A$-B-submodules of $X$. Note that $\mathcal{I}(A)$, $\mathcal{I}(B)$ and $\mathcal{S}(X)$ can be partially ordered by inclusion, and are then lattices. Then there are natural lattice isomorphisms among $\mathcal{I}(A), \mathcal{I}(B)$ and $\mathcal{S}(X)$ given by

$$
\begin{aligned}
& \mathcal{I}(A) \ni I \longmapsto{ }_{I} X \in \mathcal{S}(X), \text { where }{ }_{I} X=\left\{\left.y \in X\right|_{A}\langle y, x\rangle \in I \text { for all } x \in X\right\} ; \\
& \qquad \mathcal{S}(X) \ni Y \longmapsto I_{Y} \in \mathcal{I}(A),{ }_{Y} I \in \mathcal{I}(B), \\
& \text { where } I_{Y} \text { is the closed linear span of }\left\{{ }_{A}\langle y, x\rangle \mid y \in Y \text { and } x \in X\right\} \\
& \text { and }{ }_{Y} I \text { is the closed linear span of }\left\{\langle x, y\rangle_{B} \mid y \in Y \text { and } x \in X\right\} ; \\
& \mathcal{I}(B) \ni J \longmapsto X_{J} \in \mathcal{S}(X), \text { where } X_{J}=\left\{y \in X \mid\langle x, y\rangle_{B} \in J \text { for all } x \in X\right\} .
\end{aligned}
$$

We refer to such lattice isomorphisms among $\mathcal{I}(A), \mathcal{I}(B)$ and $\mathcal{S}(X)$ as the Rieffel correspondences (see [6, 3.3]). Note that ${ }_{I} X$ is the closed linear span of $I \cdot X$ (cf. 6, Lemma 3.23]), and we will employ this fact in the proof of Lemma 2.2.

Lemma 2.2. Let $A$ and $B$ be $C^{*}$-algebras and let $X$ be an $A$-B-imprimitivity bimodule. For any closed ideal $I$ of $A$, we have $\left({ }_{I} X\right)^{\perp}={ }_{I^{\perp}} X$. 
Proof. Since ${ }_{I} X$ is the closed linear span of $I X$, we see from ${ }_{A}\left\langle I^{\perp} X, I X\right\rangle=$ $I^{\perp} \cdot{ }_{A}\langle X, X\rangle \cdot I=\{0\}$ that ${ }_{I^{\perp}} X \subset\left({ }_{I} X\right)^{\perp}$. For the reverse inclusion, let $Y=(I X)^{\perp}$ and note that $\{0\}={ }_{A}\langle Y, I X\rangle={ }_{A}\langle Y, X\rangle \cdot I$ implies that ${ }_{A}\langle Y, Y\rangle$ is contained in $I^{\perp}$, hence $Y \subset I^{\perp} X$ by a well-known argument.

Now we are in a position to establish the main result.

Theorem 2.3. Let $A$ and $B$ be $C^{*}$-algebras and let $X$ be an $A$-B-imprimitivity bimodule. Then every closed $A$-B-submodule of $X$ is orthogonally closed in $X$ if and only if every closed $A$-B-submodule of $X$ is orthogonally complemented in $X$.

Proof. Suppose that every closed $A$ - $B$-submodule of $X$ is orthogonally closed in $X$. Let $I$ be any closed ideal of $A$. We claim that $A=I \oplus I^{\perp}$. Since it follows from Lemma 2.2 that $\left({ }_{I} X\right)^{\perp}={ }_{I^{\perp}} X$, we obtain $\left({ }_{I} X\right)^{\perp \perp}=\left({ }_{I^{\perp}} X\right)^{\perp}$, and with $I$ replaced by $I^{\perp}$ we have $\left(I^{\perp} X\right)^{\perp}={ }_{I^{\perp \perp}} X$. Since ${ }_{I} X$ is a closed $A$-B-submodule, by assumption we see that ${ }_{I^{\perp \perp}} X=\left({ }_{I^{\perp}} X\right)^{\perp}=\left({ }_{I} X\right)^{\perp \perp}={ }_{I} X$. By the Rieffel correspondence, we then see that $I^{\perp \perp}=I$. Thus we see that every closed ideal of $A$ is orthogonally closed in $A$. Hence Lemma 2.1 yields that $A=I \oplus I^{\perp}$ for any closed ideal $I$.

Let $Y$ be any closed $A$-B-submodule of $X$. Since $I_{Y}$ is a closed ideal of $A$, it follows from the above claim that $A=I_{Y} \oplus\left(I_{Y}\right)^{\perp}$. We remark that $I_{Y^{\perp}}=\left(I_{Y}\right)^{\perp}$ (see the proof of [3, Theorem 2.3] for the details). Since the Rieffel correspondences are natural lattice isomorphisms among $\mathcal{I}(A), \mathcal{I}(B)$ and $\mathcal{S}(X)$, a least upper bound $Y \vee Y^{\perp}\left(=Y \oplus Y^{\perp}\right)$ of $Y$ and $Y^{\perp}$ corresponds to a least upper bound $I_{Y} \vee I_{Y \perp}$ $\left(=I_{Y} \vee\left(I_{Y}\right)^{\perp}=I_{Y} \oplus\left(I_{Y}\right)^{\perp}=A\right)$ of $I_{Y}$ and $I_{Y^{\perp}}$. Since $A$ and $X$ correspond by the Rieffel correspondence, we conclude that $X=Y \oplus Y^{\perp}$.

We denote by $\widehat{A}$ the spectrum of $A$, that is, the set of (unitary) equivalence classes of nonzero irreducible representations of $A$ equipped with the Jacobson topology. We note that $\widehat{A}$ is a locally compact space, not necessarily a $T_{0}$-space. The reader is referred to [1] for the spectrum of a $C^{*}$-algebra.

Corollary 2.4. Let two $C^{*}$-algebras $A$ and $B$ be Morita equivalent and let $X$ be an A-B-imprimitivity bimodule. Consider the following conditions:

(1) The spectrum $\widehat{A}$ of $A$ is discrete in the Jacobson topology.

(2) The spectrum $\widehat{B}$ of $B$ is discrete in the Jacobson topology.

(3) Every closed $A$-B-submodule of $X$ is complemented in $X$.

(4) Every closed $A$-B-submodule of $X$ is orthogonally closed in $X$.

Then we have $(1) \Longleftrightarrow(2) \Longrightarrow(3) \Longleftrightarrow(4)$. If either $\widehat{A}$ or $\widehat{B}$ is a $T_{1}$-space, then conditions (1) - (4) are equivalent.

Proof. This easily follows from [3, Theorem 2.3] and Theorem 2.3 above.

Note that in the implication $(4) \Longrightarrow(1)$ above, the assumption that either $\widehat{A}$ or $\widehat{B}$ be a $T_{1}$-space is necessary (see [3, Remark $\left.2.4(2)\right]$ ). We end this paper by stating a remark.

Remark 2.5. Let $A$ and $B$ be $C^{*}$-algebras and let $X$ be an $A$-B-imprimitivity bimodule. Consider the following conditions:

(1) Every closed right $B$-submodule of $X$ is orthogonally closed in $X$.

(2) Every closed right $B$-submodule of $X$ is orthogonally complemented in $X$.

(3) Every closed $A$-B-submodule of $X$ is orthogonally closed in $X$. 
(4) Every closed $A$ - $B$-submodule of $X$ is orthogonally complemented in $X$.

Then the equivalence of (1) and (2) follows from [7], the implication (2) $\Longrightarrow(4)$ is obvious, and the equivalence of (3) and (4) is nothing but Theorem 2.3. When $B$ is simple, it follows from an easy application of the Rieffel correspondence that condition (4) is true. In this case, if $B$ is not an elementary $C^{*}$-algebra, both conditions (1) and (2) are false (see [5] and [7]), that is, condition (4) does not necessarily imply condition (2). Thus we see that $(1) \Longleftrightarrow(2) \underset{\nLeftarrow}{\rightrightarrows}(3) \Longleftrightarrow(4)$.

\section{ACKNOWLEDGEMENT}

This research was financially supported by the Kansai University Research Grants: Grant-in Aid for Encouragement for Scientists 2003.

\section{REFERENCES}

1. J. Dixmier, $C^{*}$-algebras, North Holland, New York, 1982. MF 0458185 (56:16388)

2. M. Kusuda, Three-space problems in discrete spectra of $C^{*}$-algebras and dual $C^{*}$-algebras, Proc. Royal Soc. Edinburgh 131A (2001), 701-707. MR1838507 (2002m:46087)

3. M. Kusuda, Discrete spectra of $C^{*}$-algebras and complemented submodules in Hilbert $C^{*}$ modules, Proc. Amer. Math. Soc. 131 (2003), 3075-3081. MR.1993216 (2004d:46067)

4. E. C. Lance, Hilbert $C^{*}$-modules, London Math. Soc. Lecture Note Series Vol. 210, Cambridge Univ. Press, Cambridge, 1994. MR.1325694 (96k:46100)

5. B. Magajna, Hilbert $C^{*}$-modules in which all closed submodules are complemented, Proc. Amer. Math. Soc. 125 (1997), 849-852. MR1346981 (97e:46079)

6. I. Raeburn and D. P. Williams, Morita equivalence and continuous-trace $C^{*}$-algebras, Math. Surveys Monographs Vol. 60, Amer. Math. Soc., Providence, 1998. MR 1634408 (2000c:46108)

7. J. Schweitzer, A description of Hilbert $C^{*}$-modules in which all closed submodules are orthogonally closed, Proc. Amer. Math. Soc. 127 (1999), 2123-2125. MR.1646207 (99j:46068)

Department of Mathematics, Faculty of Engineering, Kansai University, Yamate-cho 3-3-35, Suita, Osaka 564-8680, JaPAN

E-mail address: kusuda@ipcku.kansai-u.ac.jp 